13.P3

\title{
Non-Chromaffin Cell Constituents of the Adrenal Medulla are Detrimental to the Survival of Grafted Adrenal Chromaffin Cells: Studies in Rats and Non-Human Primates
}

\author{
Sherry B. Schueler, John D. Ortega, Jacqueline Sagen, and Jeffrey H. Kordower ${ }^{1}$ \\ Department of Anatomy and Cell Biology, University of Illinois School of Medicine, and ${ }^{1}$ Department of \\ Neurological Sciences, Rush Presbyterian-St. Luke's Medical Center, Chicago, Illinois 60612, USA
}

The initial rationale for using adrenal chromaffin cells in transplantation experiments was to provide a paraneural source of dopamine to replenish the dopamine insufficiency created in animal models of parkinsonism and idiopathic Parkinson's disease. Intraventricular transplants of adrenal medulla (AM) survive, synthesize and secrete catecholamines, and reverse drug-induced motor asymmetries in unilateral nigrostriatal lesioned rats. Subsequent studies in which AM grafts were placed directly into the striatum also induced functional recovery, albeit partially, and short-lived. It soon became increasingly clear that chromaffin cells survived poorly within the striatal parenchyma. Studies carried out in non-human primates and autopsy cases from AM-grafted parkinsonian patients confirmed the notion that adrenal chromaffin cells do not survive well following intrastriatal transplantation.

The use of trophic factors to improve chromaffin cell survival heralded the second era of $\mathrm{AM}$ transplantation. Injections of $\beta$ nerve growth factor into AM graft sites augments chromaffin cell survival, induces morphological differentiation, and increases the magnitude and duration of functional effects. Chromaffin cell survival can also be increased by cografting AM with growth factor-producing C6 gliomas, astrocytes genetically engineered to produce $\beta$ NGF, and transected peripheral nerve whose damaged Schwann cells secrete a variety of trophic molecules including $\beta$ NGF.

We have begun to utilize an alternative approach towards enhancing chromaffin cell survival. Instead of using trophic molecules to enhance graft viability we have attempted to minimize factors which may negatively impact upon chromaffin cell survival and induce AM grafts to degenerate. Adrenal chromaffin cells have previously been demonstrated to survive well following implantation into the periaqueductal gray once the chromaffin cells were isolated from nonchromaffin cells found in the AM. These data suggest that fibroblasts, blood-borne leukocytes, and/or endothelial cells within the AM may be detrimental to chromaffin cell viability and, at least in part, underlie the poor survival of these cells following intrastriatal implantation.

We presently assessed the effects of isolating bovine chromaffin cells from the other cell types within the AM upon chromaffin cell graft survival in rats and MPTP treated rhesus monkeys following intrastriatal transplantation. Three groups of immunosuppressed rats were employed. Group 1 received grafts of bovine AM following gland perfusion and dissection of the medulla from the adrenal cortex. Group 2 received implants of isolated adrenal chromaffin cells. Chromaffin cells were isolated in vitro by placing them in a Percoll gradient resulting in the segregation of dead cells, residual cortical cells, red blood cells, and viable medullary cells. Once separated, the medullary cells were differentially plated resulting in a $95 \%$ pure population of chromaffin cells. Group 3 received implants of isolated adrenal chromaffin cells which were reseeded with fibroblasts and endothelial cells which were allowed to proliferate in culture. All rats were sacrificed 1-2 months following transplantation. Rats in Group 1 displayed viable tyrosine hydroxylase $(\mathrm{TH})$ and dopamine $\beta$ hydroxylase (D $\beta \mathrm{H})$-immunoreactive (ir) transplants. However, these grafts tended to be small, the graft-host interface was infiltrated with macrophages, and many of the chromaffin cells appeared to be in the process of degenera- 
tion. In contrast, rats receiving implants of isolated chromaffin cells displayed very large and healthy appearing $\mathrm{TH}$-ir and $\mathrm{D} \beta \mathrm{H}$-ir transplants which retained their endocrine phenotype. Rats in this group displayed a 2 - and 3-fold increase in surviving chromaffin cells one and two months following transplantation, respectively, relative to rats in Group 1. Isolated chromaffin cell implants appeared to integrate well within the host and few macrophages were evident. The reseeding of isolated chromaffin cells with fibroblasts and endothelial cells impaired chromaffin cell survival. Most rats in this group failed to display surviving implants and the few surviving chromaffin cells appeared to be degenerating. Interestingly, the perigraft region in rats from this group displayed a dense $\mathrm{TH}$, but not $\mathrm{D} \beta \mathrm{H}$, fiber network, suggesting that signals from degenerating implants may modify the host nigrostriatal system.
To assess the ability of isolated adrenal chromaffin cells to survive grafting in the primate brain, these cells were implanted into the caudate and putamen of immunosuppressed hemiparkinsonian rhesus monkeys. One month following transplantation, robust survival $(>1,000,000)$ of endocrine appearing TH- and $\mathrm{D} \beta \mathrm{H}$-immunoreactive chromaffin cells was observed. These cells appeared to integrate well within the striatum.

These data strongly suggest that non-chromaffin cell constituents within the AM are detrimental to the survival of chromaffin cells following intracerebral transplantation. Isolating chromaffin cells prior to transplantation may significantly enhance their survival and may be an improved donor source for the treatment of Parkinson's disease.

Supported by UPF and NS28931. 

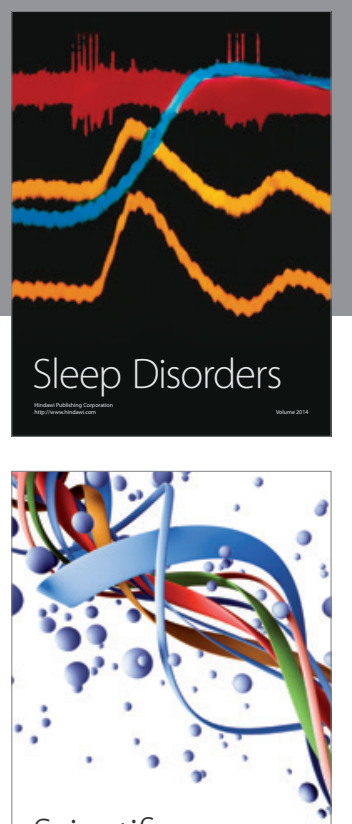

Scientifica
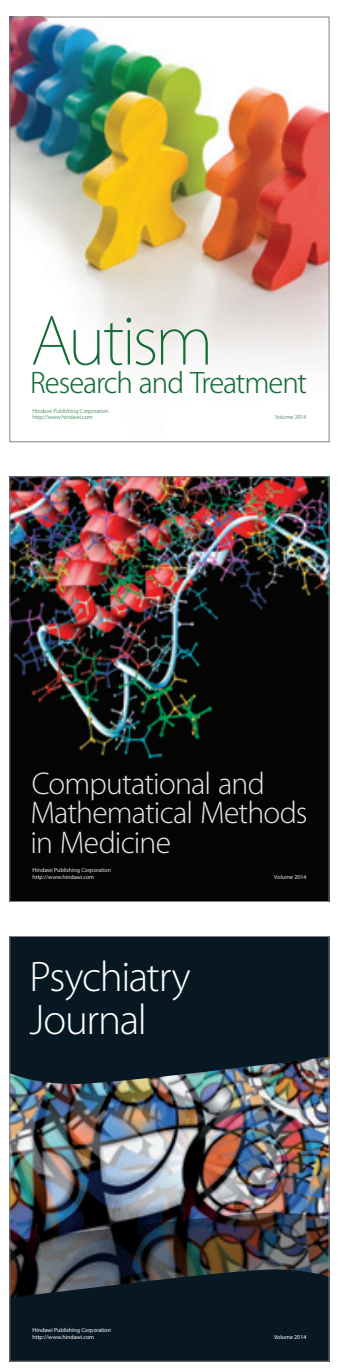
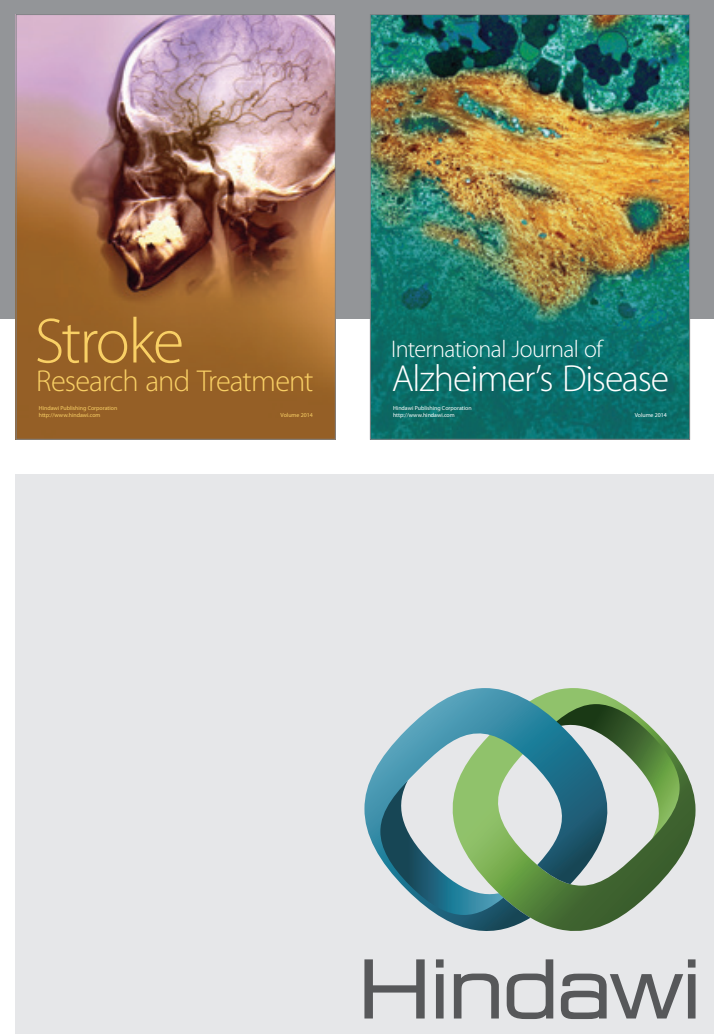

Submit your manuscripts at

http://www.hindawi.com
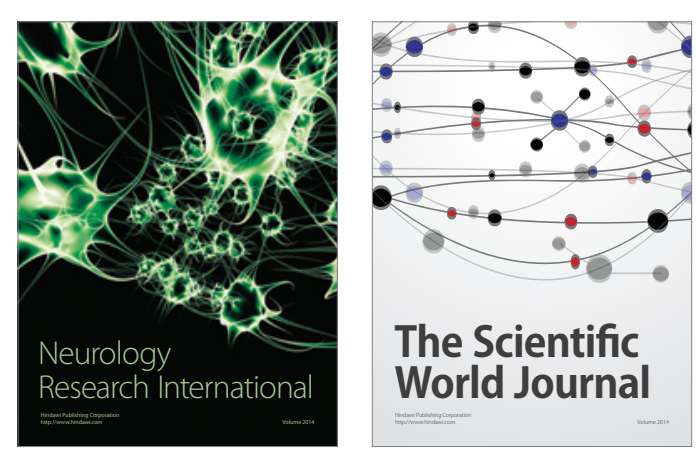

The Scientific World Journal

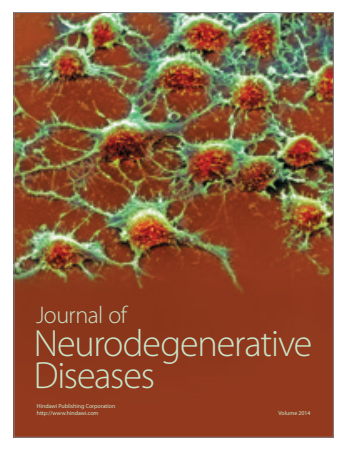

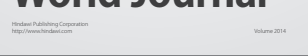

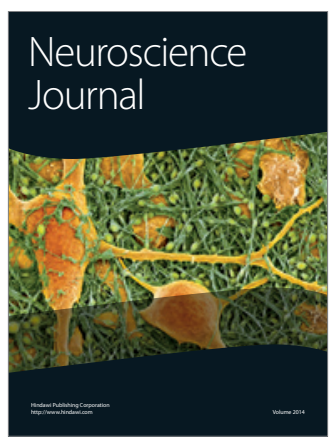

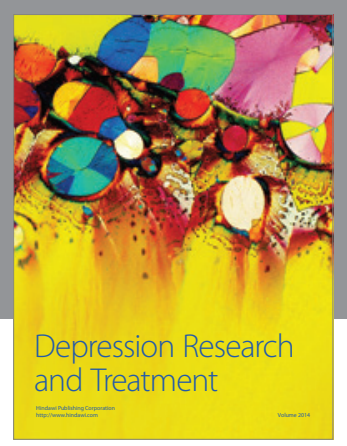
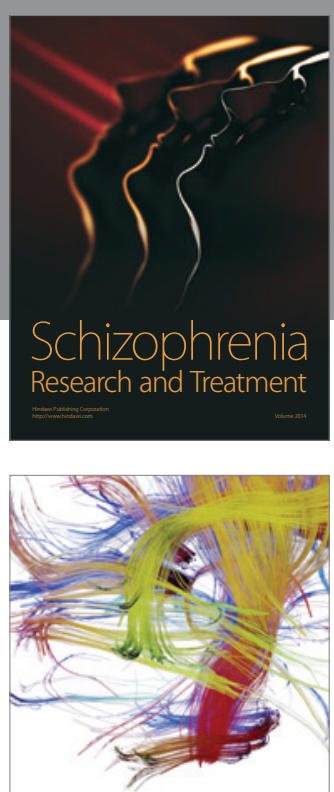

Brain Science

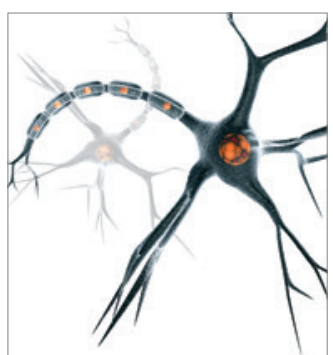

Neural Plasticity
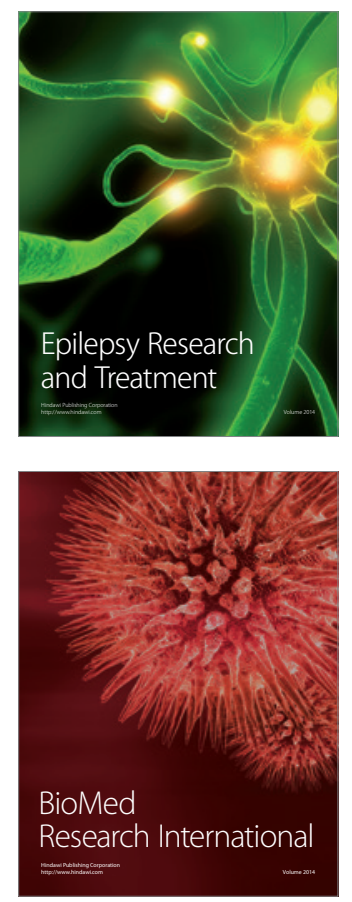

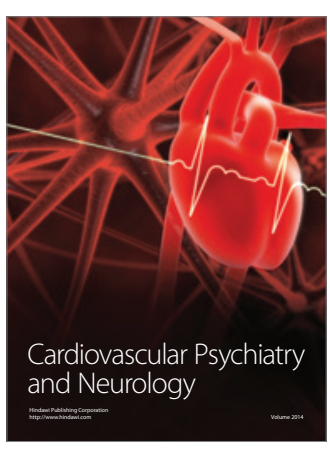

Parkinson's

Disease
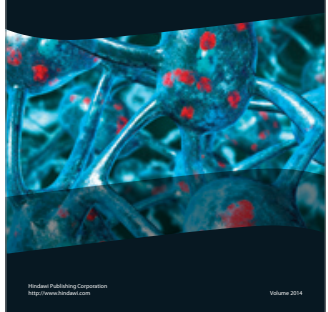